
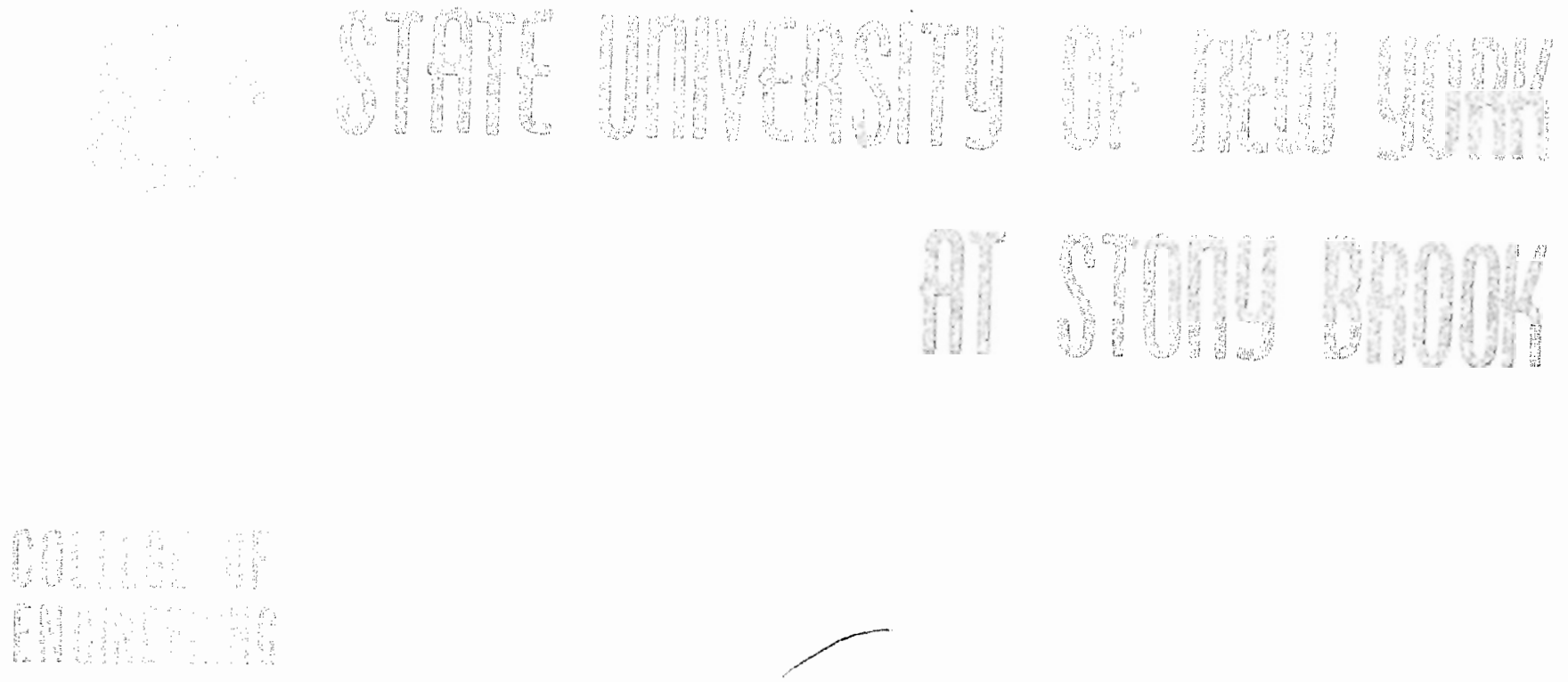

Report No. 7

THE INFLUENCE OF FINITE WALL CONDUCTANCE

ON THE LOAD CAPACITY OF THE MAGNETOHYDRODYNAMIC SLIDER BEARING

by

Wi11iam T. Sryder

OCTOBER 1963 
W. T. SNYDER

Associate Professor of Engineering, Department of Thermal Sciences and

Fluid Mechanics,

State University of New York, Stony Brook, N. Y.

\section{on Load Capacity of the Magnetohydrodynamic Slider Bearing}

An analysis is presented which considers the infuence of finite-wall conductance on the pressure distribution and load capacity of the magnetohydrodynamic slider bearing. The analysis is based on general external loading conditions with the open-circuit condition being a special case. The load capacity is a linear function of the quantity $\Phi=E_{z} / V B_{y}$ and the dependence of $\Phi$ on the conductivity and thickness of the walls is shown in explicit form. Curves showing the variation of $\Phi$ with wall conductance are presented. A numerical example is included which indicates a substantial reduction of load capacity from the case of insulating walls to the case where the wall conductivity is 1 percent of the fluid conductivity.

\section{Introduction}

IN recent years, a considerable interest has been shown in the potentialities of liquid metals as lubricants $[1,2] .{ }^{1}$ Liquid metals may be utilized under extreme conditions of lubrication at which conventional lubricants would experience undesirable physicochemical changes. The relatively high thermal conductivity of liquid metals means that the heat generated in the film because of viscous dissipation is readily conducted away from the source of generation, resulting in a tendency toward uniformity of temperature and viscosity within the lubricant film.

Because of the large electrical conductivity of liquid metals, the possibility of electromagnetic pressurization from the application of an external magnetic field arises. Within the past two years, an exploitation of the possibilities of magnetohydrodynamic (MHD) lubrication has begun [3 to 8]. The work to date has been analytical with no experimental data reported in the open literature to the author's knowledge. The analytical studies have served to demonstrate theoretically the possibility of increasing the load capacity of liquid-metal lubricants by means of an applied magnetic field. The qualitative and quantitative features of

\footnotetext{
1 Numbers in brackets designate References at end of paper.

Contributed by the Lubrication Division of THE AMERICAN SoCIETY of Mechanical Enginemers for presentation at the ASLEASME Lubrication Conference, Rochester, N. Y., October 15-17, 1963. Manuscript received at ASME Headquarters, July 8, 1963. I'aper No. 63-Lub-4.
}

various geometric and electromagnetic-field configurations have been investigated to such an extent that MHD lubrication has become rather firmly established as a research area in hydrodynamic lubrication.

The present paper represents an attempt to extend the state of knowledge of MHD lubrication by considering one aspect of the problem which has not been considered before. In this paper, the influence of the properties of the walls comprising the bearing and slider surfaces is investigated. In particular, the effect of the electrical conductivity and thickness of these walls on the pressure distribution is considered. For this purpose, the linear, inclined slider bearing is used. As shown in reference [6], the load capacity of the MHD bearing can be improved by applying an external electric field to augment the electric field induced by the motion of the conducting fluid through the magnetic field. In the present anajysis, an externally applied electric field is utilized. The open-circuit and short-circuit conditions then become special cases of the case of general external loading. In reference [9], the wall properties were shown to have a significant effect on the velocity distribution in $\mathrm{MHD}$ channel flow, and from this result, one would anticipate a strong dependence of the load capacity on the wall properties in MHD lubrication.

\section{The Analysis \\ Equation of Motion}

The slider-bearing geometry is shown in Fig. 1. The properties of the lower, moving wall are identified by the subscript 1

$$
\begin{aligned}
& \text { Nomenclature. } \\
& b=w_{1} / w_{0} \\
& B_{y}=\text { applied magnetic field in } \\
& \theta_{2}=\frac{\sigma_{2} h_{2}}{\sigma W_{0}} \\
& \mu=\text { viscosity } \\
& \xi=y / W_{0} \\
& \pi=\left(P / \frac{\mu V}{W_{0}}\right) \\
& \pi_{0}=\text { value of } \pi \text { at } X=0 \text { and } \\
& X=L \\
& \rho=\text { fluid density } \\
& \sigma=\text { fluid electrical conductivity } \\
& \sigma_{1}=\text { lower wall electrical conduc- } \\
& \sigma_{2}=\text { upper wall electrical conduc- } \\
& \Phi=E_{z} / V B y \\
& \Phi_{\text {ins }}=\underset{\text { walls }}{\text { value for }} \Phi \text { for insulating }
\end{aligned}
$$


$\mathrm{B}_{\mathrm{Y}}$

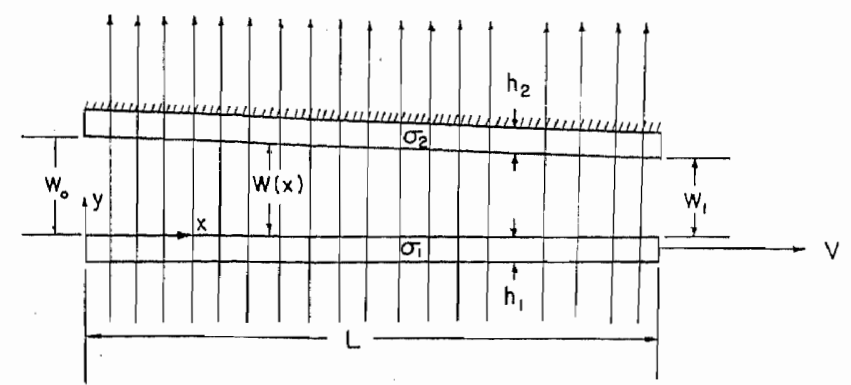

Fig. 1 MHD slider-bearing geometry

and those of the upper, stationary wall by subscript 2 . The lower wall moves with a constant velocity $V$. The fluid properties will be assumed constant so that the energy equation is not needed in the anaiysis. The bearing is considered infinite in the $z$-direction and a uniform magnetic field is applied in the $y$-direction.

The governing equation of motion has been discussed in reference [3] and may be written as

$$
-\frac{d P}{d x}+\mu \frac{\partial^{2} u}{\partial y^{2}}-J_{z} B_{y}=0
$$

The electric current is given by the simple form of Ohm's law (neglecting the Hall effect):

$$
J_{z}=\sigma\left(E_{z}+u B_{y}\right)
$$

It is convenient to define the following dimensionless parameters:

$$
\gamma=\frac{u}{V}, \quad \eta=\frac{W}{W_{0}}, \quad \xi=\frac{y}{W_{0}}, \quad \alpha=\frac{x}{W_{0}}, \quad \pi=\frac{P}{\frac{\mu V}{W_{0}}}
$$

In terms of the dimensionless parameters, the equation of motion becomes

$$
\frac{\partial^{2} \gamma}{\partial \xi^{2}}-M^{2} \gamma=\frac{d \pi}{d \alpha}+M^{2} \Phi
$$

where

$$
\begin{aligned}
M & =W_{0} B_{u}\left(\frac{\sigma}{\mu}\right)^{1 / 2}=\text { Hartmann number } \\
\Phi & =\frac{E_{z}}{V B_{y}}=\text { dimensionless electric field }
\end{aligned}
$$

It is shown in reference [3] that the electric field $E_{z}$ is constant.

Equation (4) may be integrated across the film since $\pi$ is assumed constant across the film. The appropriate nonslip boundary conditions are

$$
\gamma(\xi=0)=1 \quad \gamma(\xi=\eta)=0
$$

The solution to equation (4) subject to the boundary conditions of (5) may be written in the form

$$
\begin{aligned}
\gamma= & \left\{\frac{1}{M^{2}} \frac{d \pi}{d \alpha}+\Phi\right\} \\
& =\left\{\frac{\sinh M \eta(\cosh M \xi-1)-\sinh M \xi(\cosh M \eta-1)}{\sinh M \eta}\right\} \\
& +\left\{\frac{\sinh M \eta \cosh M \xi-\sinh M \xi \cosh M \eta}{\sinh M \eta}\right\}
\end{aligned}
$$

The velocity solution depends on the two electromagnetic parameters, $M$ and $\Phi$, the latter determined by the external electrical loading conditions.
The continuity condition is satisfied by the requirement that the total mass flow across each section be constant, a condition expressed by

$$
m=\int_{0}^{W} \rho u d y=\rho V w_{0} \int_{0}^{\eta} \gamma d \xi
$$

Substituting equation (6) into (7) and performing the integration gives

$$
M\left(\frac{m}{\rho V W_{0}}\right) G(\mathbf{M} \eta)=1+\left\{\frac{1}{\mathbf{M}^{2}} \frac{d \pi}{d \alpha}+\Phi\right\} F(\mathbf{M} \eta)
$$

where

$$
\begin{gathered}
F(M \eta)=2-M \eta \operatorname{ctnh} \frac{M \eta}{2} \\
G(M \eta)=\operatorname{ctnh} \frac{M \eta}{2}
\end{gathered}
$$

The pressure distribution is determined by solving equation (8) for $d \pi / d \alpha$ and integrating over the length of the bearing. For this purpose, it is convenient to express $\pi$ in terms of $\eta$ by the transformation

$$
\frac{d}{d \alpha}=\frac{d}{d \eta} \frac{d \eta}{d \alpha}=-\frac{W_{0}-W_{1}}{L} \frac{d}{d \eta}
$$

Solving for the pressure gradient from equation (8) gives

$$
\frac{W_{0}-W_{1}}{\mathbf{M}^{2} L} \frac{d \pi}{d \eta}=\frac{1}{F(\mathbf{M} \eta)}-\mathbf{M}\left(\frac{m}{\rho V W_{0}}\right) \frac{G(\mathbf{M} \eta)}{F(\mathbf{M} \eta)}+\Phi
$$

The boundary conditions to be satisfied by $\pi$ are

$$
\pi(\eta=1)=\pi_{0} \quad \pi(\eta=b)=\pi_{i}
$$

Integrating equation (9) subject to the boundary conditions of (10) and introducing additional notation gives for the pressure distribution

$$
\frac{W_{0}-W_{1}}{L}\left(\pi-\pi_{0}\right)=M^{2}\{R(\mathbf{M} \eta)+\Phi(1-b) S(\mathbf{M} \eta)\}
$$

where

$$
\begin{gathered}
R(\mathbf{M} \eta)=\int_{1}^{\eta} \frac{d \eta}{F(\mathrm{M} \eta)}-\int_{1}^{b} \frac{d \eta}{F(\mathrm{M} \eta)} \frac{\int_{1}^{\eta} \frac{G(\mathrm{M} \eta)}{F(\mathrm{M} \eta)} d \eta}{\int_{1}^{b} \frac{G(\mathrm{M} \eta)}{F(\mathrm{M} \eta)} d \eta} \\
S(\mathrm{M} \eta)=\frac{\int_{1}^{\eta} \frac{G(\mathrm{M} \eta)}{F(\mathrm{M} \eta)} d \eta}{\int_{1}^{b} \frac{G(\mathrm{M} \eta)}{F(\mathrm{M} \eta)} d \eta}-\frac{1-\eta}{1-b}
\end{gathered}
$$

The load capacity is obtained by integrating equation (11) over the bearing length. The dimensionless load then becomes

$$
\begin{aligned}
\mathscr{L}= & \frac{W_{0}-W_{1}}{L} \int_{1}^{b}\left(\pi-\pi_{0}\right) d \eta \\
& =M^{2}\left\{\int_{1}^{b} R(\mathrm{M} \eta) d \eta+\Phi(1-b) \int_{1}^{b} S(\mathrm{M} \eta) d \eta\right\}
\end{aligned}
$$

It is interesting to observe that the pressure distribution and the load are linear functions of $\Phi$ for a fixed Hartmann number. It is clear that for a given value of $M$ the load capacity may be increased or decreased through $\Phi$ depending on the direction of the applied electric field. This result was pointed out by Hughes [6] for the MHD finite-step slider bearing. The wall properties will influence the magnitude of $\Phi$, and the magnitude of the 
effect of the wall properties on $\Phi$ is determined from the total current equation which will be presented in the next section.

\section{The Current Equation}

The electromagnetic boundary conditions at the interface between the fluid and the walls are discussed in Appendix A of reference [3]. In that reference, only the open-circuit and insulating-wall conditions were used. Both of these conditions will be relaxed in the present analysis.

The boundary conditions require that $E_{z}$ be continuous across the fluid-wall interface. Since $E_{z}$ is constant in the fluid, it will have the same constant value in the two walls. The total electric current flowing in the $z$-direction is then the sum of the contributions from the two walls and from the fluid. From Ohm's law, the total current expression is

$$
\begin{aligned}
I=\int_{0}^{L}\left\{\sigma_{1} \int_{-h_{1}}^{0}\left(E_{z}+V B_{y}\right) d y\right. & +\sigma \int_{0}^{W}\left(E_{z}+u B_{y}\right) d y \\
& \left.+\sigma_{2} \int_{W}^{W+h 2} E_{z} d y\right\} d x
\end{aligned}
$$

Introducing the velocity distribution from equation (6) into (13) and performing the integration gives the following expression for the dimensionless total current:

$$
I^{*}=\frac{m}{\rho V W_{0}}+\theta_{1}+\Phi\left(\frac{1+b}{2}+\theta_{1}+\theta_{2}\right)
$$

where

$$
\begin{gathered}
I^{*}=\frac{I}{\sigma L W_{0} V B_{y}} \\
\theta_{1}=\frac{\sigma_{1} h_{1}}{\sigma W_{0}} \\
\theta_{2}=\frac{\sigma_{2} h_{2}}{\sigma W_{0}}
\end{gathered}
$$

The quantity $m / \rho V W_{0}$ is determined from the integration of equation (9) subject to the boundary conditions of (10) with the result,

$$
\frac{m}{\rho V W_{0}}=\frac{1}{M} \frac{\int_{1}^{b} \frac{d \eta}{F(\mathrm{M} \eta)}-\Phi(1-b)}{\int_{1}^{b} \frac{G(\mathrm{M} \eta)}{F(\mathrm{M} \eta)} d \eta}
$$

Equation (14) with $m / \rho V W_{0}$ given by (15) then gives a single equation relating $I^{*}$ and $\Phi$ which involves the parameters $M, \theta_{1}$, $\theta_{2}$, and $b$. Either the quantity $I^{*}$ or $\Phi$ may be considered as the independent externally applied electromagnetic parameter in addition to $M$ and equation (14) then serves to determine the other parameter. Since $\Phi$ enters explicitly in the pressure distribution and load capacity expressions given by equations (11) and (12), respectively, it is convenient to solve equation (14) explicitly for $\Phi$. The result is

$$
\Phi=\frac{M\left(I^{*}-\theta_{1}\right) \int_{1}^{b} \frac{G(\mathrm{M} \eta)}{F(\mathrm{M} \eta)} d \eta-\int_{1}^{b} \frac{d \eta}{F(\mathrm{M} \eta)}}{M\left(\theta_{1}+\theta_{2}+\frac{1+b}{2}\right)}
$$

Equation (16) shows explicitly the dependence of $\Phi$ on the wall properties through the conductance ratios $\theta_{1}$ and $\theta_{2}$. For opencircuit conditions, $I^{*}=0$ and the open-circuit field value is determined from equation (16) with $I^{*}=0$. The short-circuit case corresponds to $\Phi=0$, and setting the numerator of equation (16) equal to zero determines the value of the short-circuit current.

\section{Results}

Pressure-distribution curves are calculated from equation (11) and plotted in Figs. 2, 3, 4, and 5. In Fig. 2, pressure distributions are shown for three values of $M$ for the conditions of open circuit and perfectly insulating walls. The quantity $\Phi$ is not a strong function of $M$ under these conditions. In particular, $\Phi$ has the values $-0.444,-0.436$, and -0.413 for $M$-values of 1,5 , and 10 , respectively, for $I^{*}=0$ and $\theta_{1}=0=\theta_{2}$. Shown also for comparison is the $M=0$ curve for the classical case in the absence of a magnetic field.

Figs. 3, 4, and 5 show pressure distributions for three values of $M$ with $\Phi$ as the parameter. The increase in load capacity with increasing negative values of $\Phi$ is clearly shown. It can be shown that for $\Phi=0$, corresponding to the short-circuit case, an adverse affect on pressure distribution is achieved; that is, the load capacity under short-circuit conditions is less than the classical Inad capacity of the slider bearing without a magnetic field. This result is in agreement with the result obtained by Hughes $[6]$ for the finite-step slider bearing with transverse magnetje field. Operating with positive values of $\Phi$ would correspond to the accelerating mode which is clearly detrimental from the point of view of MHD pressurization. It is therefore necessary to operate at negative values of $\Phi$ to increase the load capacity.

The influence of the wall properties on the load capacity can be determined through the dependence of $\Phi$ on wall properties as given by equation (16). Fixing $M$ and $I^{*}$ gives $\Phi$ as a function of the wall conductances $\theta_{1}$ and $\theta_{2}$. A reasonable choice for $I^{*}$ can be determined as follows:

Assuming $\theta_{1}=0=\theta_{2}$, corresponding to perfectly insulating boundaries, gives a relationship between $I^{*}$ and $\Phi$. Solving this equation for $I^{*}$ and substituting into equation (16) gives an expression for $\Phi$ including the effect of finite-conducting walls based on the same total current for insulating and conducting walls. The result can be written as

$$
\frac{\Phi}{\Phi_{\mathrm{ins}}}=\frac{M\left(\frac{1+b}{2}-\frac{\theta_{1}}{\Phi_{\mathrm{ins}}}\right) \int_{1}^{b} \frac{G(\mathrm{M} \eta)}{F(\mathrm{M} \eta)} d \eta-(1-b)}{M\left(\frac{1+b}{2}+\theta_{1}+\theta_{2}\right)}
$$

Since it has been established that favorable MHD pressurization results only if $\Phi<0$, the quantity $-\theta_{1} / \Phi_{\text {ins }}$ may be written as $+\left(\theta_{1} /\left|\Phi_{\text {ins }}\right|\right)$. Also a reduction in the value of $|\Phi|$ results in lower pressures and consequently a dearease in load capacity. Therefore for a fixed value of $M$ and $I^{*}$, a decrease in load capacity will occur owing to conducting walls if the ratio given by equation ( 17$)$ is less than one. The criterion for this to occur is

$$
\frac{\Phi}{\Phi_{\text {ins }}}<1 \quad \text { if } \quad \frac{\theta_{1}}{\left|\Phi_{\text {ins }}\right|}<\left(\theta_{1}+\theta_{2}\right)
$$

or

$$
\frac{1}{\left|\Phi_{\mathrm{ins}}\right|}<1+\frac{\theta_{2}}{\theta_{1}}
$$

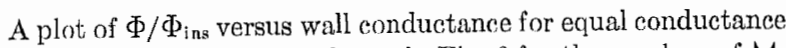
of upper and lower walls is shown in Fig. 6 for three values of $M$. The curves are based on the value $\Phi_{\text {ins }}=-10$. To cover the range of $\theta$ from 0 to $\infty$ necessitates the use of a logarithmic scale on the abscissa. The values $\theta_{\mathrm{t}}=0=\theta_{2}$ correspond to insulated surfaces whereas $\theta_{1}=\infty=\theta_{2}$ correspond to perfectly conducting surfaces which results in the short-circuit operating condition. For fixed values of wall conductances, the reduction in $|\Phi|$ below the $\left|\Phi_{\text {ins }}\right|$-value is seen to increase with $M$.

Referring to the definitions of conductances given by equations (14b) and (14c), a reasonable order-of-magnitude estimate of $h / W_{0}$ would be $h / W_{0}=0\left(10^{2}\right)$. For a $\theta$-value of 1 , the ratio of wall-to-fluid conductivity would then be of the order $10^{-2}$. Fig. 6 shows that for these conditions, $|\Phi|$ is reduced by about 70 per- 


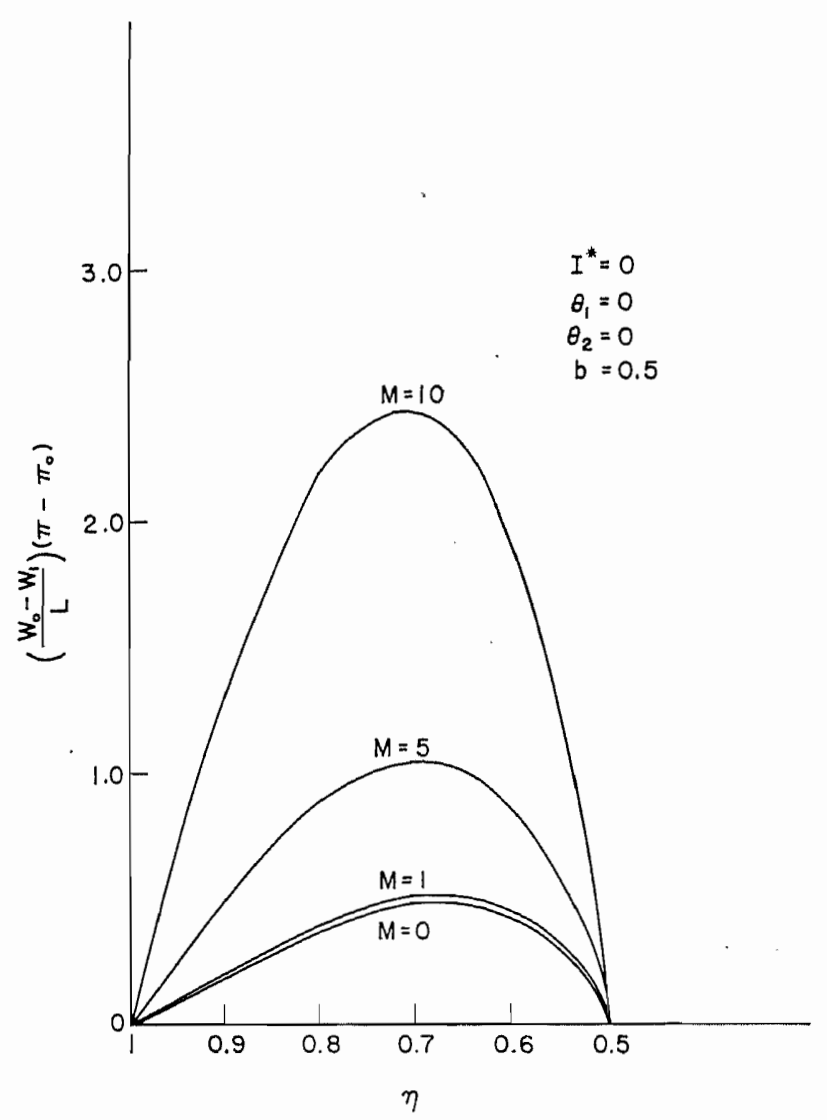

Fig. 2 Pressure distribution for conditions of open circuit and perfectly insulating walls

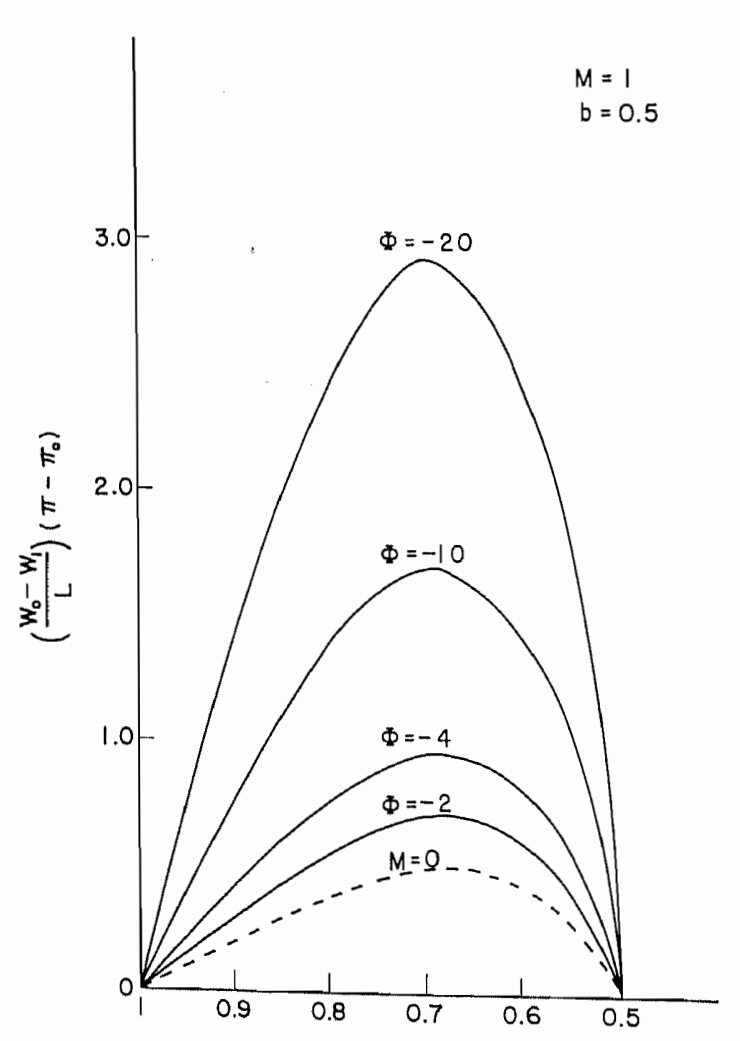

Fig. 3 Pressure distribution for $M=1$

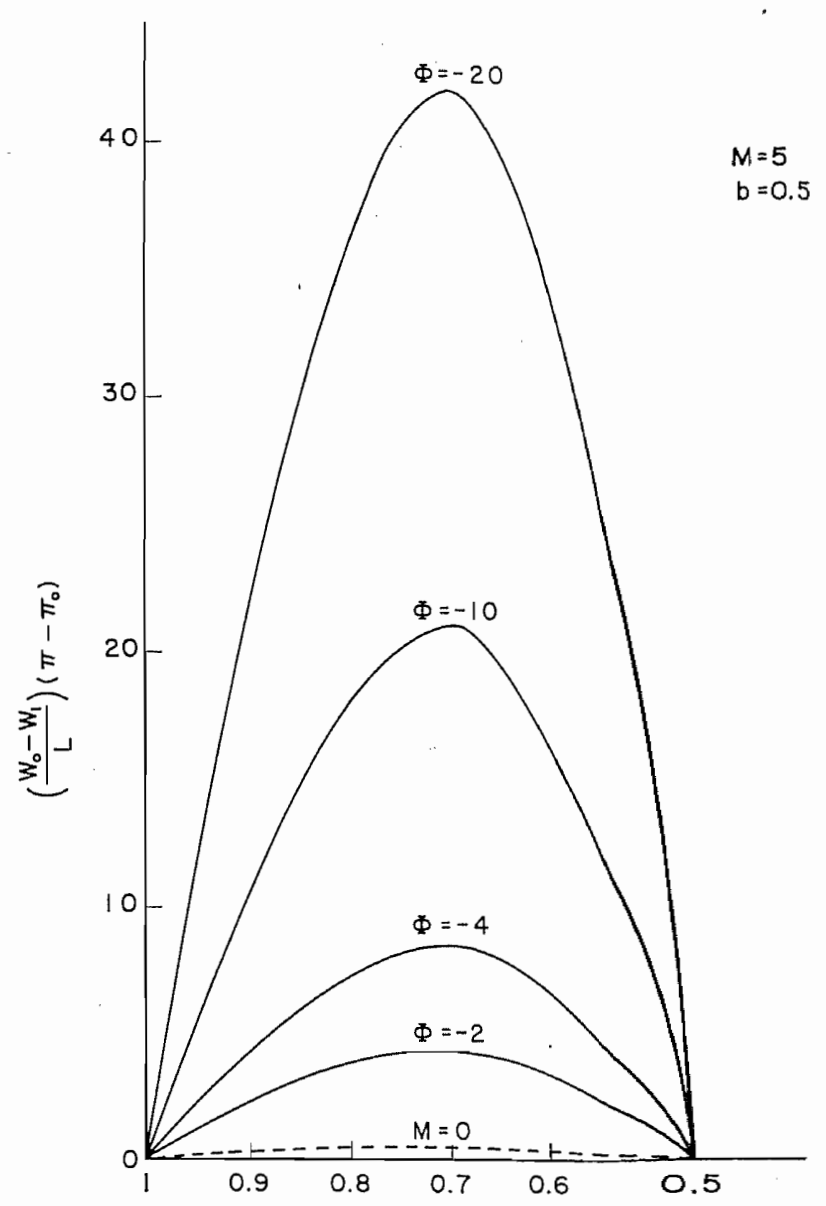

$\eta$

Fig. 4 Pressure disfribution for $M=5$

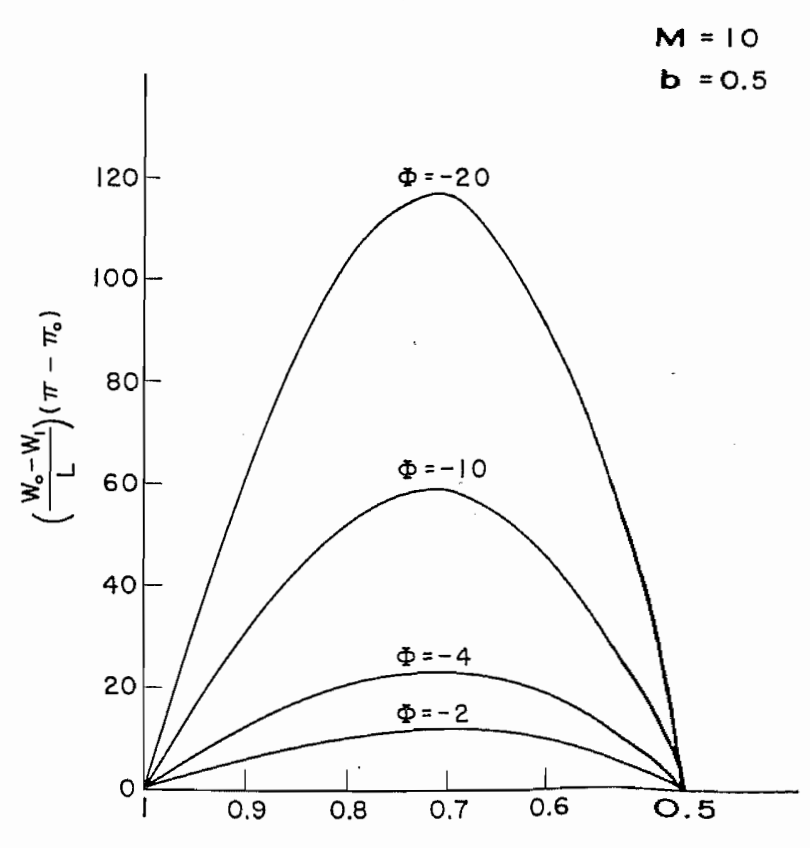

$\eta$

Fig. 5 Pressure distribution for $M=10$ 


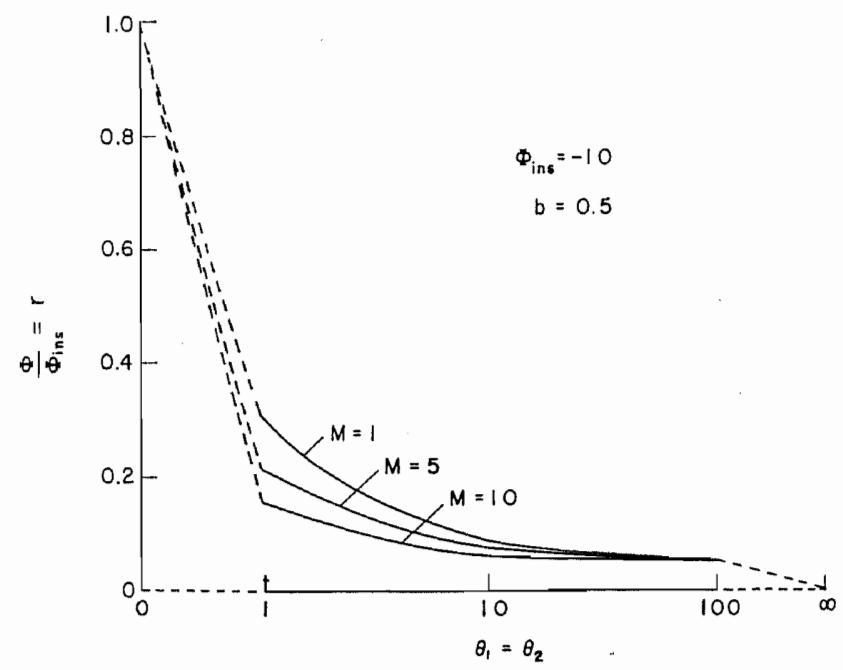

Fig. 6 Voltage ratio versus wall conductance

cent at $M=1$ and by about 85 percent at $M=10$. The implieation of this conduction on load capacity will be discussed in a particular example in the next section.

\section{Numerical Example}

Using equation (12), the dimensionless load was determined to be 18.928 for $b=0.5, M=10$, and $\Phi=-10$. The integrals involved in the functions $R(\mathrm{M} \eta), S(\mathrm{M} \eta)$, and $\&$ were evaluated numerically. Considering $\Phi=-10$ to correspond to insulated walls, the value of $\Phi$ for $\theta_{1}=1=\theta_{2}$ was determined from Fig. 6 to be -1.52 which gives a dimensionless load of 2.882 . A reduction of load capacity of 84.7 percent thus results when the walls are changed from perfect insulators to conductors with conductance ratios of 1 . This corresponds to a ratio of wall conductivity to fluid conductivity of approximately $10^{-2}$ as shown in the preceding section.

\section{References}

1 P. H. McDonald, Lubrication Behavior of Liquid Metals, NADC Contract No. AF 33(616)-5885 Progress Reports 4 and 5, 1960 .

2 W. T. Snyder and L. N. Connor, "A Journal Bearing Analysis With Application to Liquid Metal Lubricants," ASME Paper No. $61-$ WA-83.

3 W. T. Snyder, "The Magnetohydrodynamic Slider Bearing," Journal of Basic Engineming, Trans. ASME, Series D, vol. 84, 1962, p. 197.

4 W. F. Hughes and R. A. Elco, "Magnetohydrodynamic Lubrication Flow Between Parallel Rotating Disks," Journal of Fluid Mechanics, vol. 13, part 1, 1962, p. 21.

5 W. F. Hughes and R. A. Elco, "The Magnetohydrodynamic Journal Bearing," Journal of American Rocket Society, vol. 32, 1962 , p. 776 .

6 W. F. Hughes, "The Magnetohydrodynamic Finite-Step Slider Bearing," Journal of Basic Enginedring, Trans. ASME, Series D, vol. 85, 1963, pp. 129-136.

7 D. C. Kuzma, "The Magnetohydrodynamic Journal Bearing," ASME Paper No. 63-Lub-16 to be published.

8 W. Fucks and J. Uhlenbusch, "Magnetohydrodynamic Theory of Lubrication." The Physics of Fluids, vol. 5, 1962, p. 498.

9 C. C. Chang and J. T. Yen, "Magnetohydrodynamic Channel Flow as Influenced by Wall Conductance," Zeitschrift für angewandte Mathematili und Physik, vol. 13, 1962, p. 266. 